\title{
Educación Física y Salud. Evaluación de la aptitud muscular y la adiposidad corporal en adolescentes escolares
}

\author{
Physical Education and Health. Evaluation of muscular fitness and body \\ fat in school adolescents
}

* Pablo Ariel Torres

Torres, P. (2021). Educación Física y Salud. Evaluación de la aptitud muscular y la adiposidad corporal en adolescentes escolares. Revista Ciencias de la Actividad Física UCM, 22(2), julio-diciembre, 1-12. https://doi.org/10.29035/rcaf.22.2.5

\section{RESUMEN}

La Educación Física, como asignatura escolar obligatoria, debe realizar acciones para hacer frente a los altos niveles de inactividad física, sedentarismo y obesidad en la población, destacándose en importancia, la evaluación de la aptitud física y su posterior programación del ejercicio en las escuelas. Los propósitos del estudio fueron: 1) Evaluar de manera sencilla la condición física de un curso del nivel escolar secundario ( $\mathrm{n}=17$ ), de un colegio privado de la ciudad de La Plata, provincia de Buenos Aires, Argentina. 2) Conocer y relacionar los niveles de aptitud muscular y adiposidad corporal en los alumnos. Tras la investigación, se conoció que la obesidad influye negativamente en los resultados de las evaluaciones de la aptitud muscular, como muestran otros estudios. Se pudo concluir que es posible la evaluación de la condición física de los escolares para la obtención de información valiosa en pos de la prevención de la inactividad física.

Palabras clave: Educación Física; salud; aptitud muscular; adiposidad.

\begin{abstract}
Physical Education, as a compulsory school subject, must take actions to deal with the high levels of physical inactivity, sedentary lifestyle and obesity in the population. Standing out in importance, the evaluation of physical fitness and its subsequent programming of exercise in schools. The purposes of the study were: 1) To evaluate in a simple way the physical condition of a course at the secondary school level $(n=17)$, of a private school in the city of La Plata, province of Buenos Aires, Argentina. 2) Know and relate the levels of muscle fitness and body fat in students. After the investigation it was known that obesity negatively influences the results of evaluations of muscular fitness, as other studies show. Concluding that it was possible to assess the physical condition of school students, in order to obtain valuable information in order to prevent physical inactivity.
\end{abstract}

Key words: Physical Education; muscle fitness health; adiposity. 


\section{INTRODUCCIÓN}

La Educación Física (EF), como asignatura escolar obligatoria, debe realizar acciones para hacer frente a los altos niveles de inactividad física, sedentarismo y obesidad en la población. Esta situación preocupante es tratada por la Organización Mundial de la Salud (World Health Organization, 2010), quien reconoce como epidemia mundial del siglo XXI al sedentarismo (tabaquismo, alcoholismo y las dietas malsanas), identificándolo como uno de los factores de riesgo de las enfermedades no transmisibles (diabetes, cáncer, enfermedades crónicas respiratorias y cardiovasculares). Debido a que la reducción de actividad física (AF) es crítica por su implicancia negativa para el crecimiento, desarrollo, bienestar, salud y estado físico de niños y jóvenes (Malina, 2010), es necesario tomar medidas idóneas para dar respuestas al problema.

En este contexto, se concibe a la AF regular como el mecanismo para la prevención primaria, por sus beneficios relacionados al mantenimiento del peso, la adiposidad corporal (Ministerio de salud de la Nación Argentina, 2013), la presión arterial normal, la salud esquelética, la aptitud aeróbica, la fuerza y resistencia muscular (Carnero et al., 2015, Malina, 2010). Se ha demostrado que la participación en la AF regular reduce el riesgo de la enfermedad coronaria y del accidente cerebrovascular, diabetes, hipertensión, cáncer de colon, cáncer de mama y depresión (World Health Organization, 2010). Además, es interesante destacar los beneficios que trae aparejado el ejercicio físico regular para los jóvenes como: la prevención del tabaquismo, alcoholismo y el consumo de otras sustancias perjudiciales (Nikolić et al., 2020).

Para los niños y adolescentes, lo recomendable por expertos de Estados Unidos, Australia y Reino Unido, es la realización de AF moderada durante 60 minutos diarios, tanto de tipo aeróbica, como de fortalecimiento muscular y óseo. Este mayor tiempo de actividad (en comparación con las guías para adultos), se debe a que en estas tempranas edades se necesitan mayores requerimientos en pos del desarrollo de diferentes habilidades motoras, claves para un estilo de vida activa en la adultez. Como así también, la promoción de la salud y la reducción del riesgo de enfermedades crónicas (US Dept of Health and Human Services, 2008).

En esta línea, el 45,4\% de la población urbana Argentina entre 5 y 17 años (escolares) no logra cumplir con las recomendaciones previas sobre la realización de AF. De esos niños y jóvenes, el 61,8\% son propensos al comportamiento sedentario, lo que incrementa el riesgo para la salud (Tuñon \& Laíño, 2014). Esta situación, que sobrepasa fronteras, es definida por Pradenas et al. (2017) como un fenómeno cultural preocupante, sobre el cual hay que enfocarse y proponer actividades de prevención primaria e impactar en los comportamientos colectivos de los agentes de salud, funcionarios gubernamentales y administradores escolares, entre otros (Faigenbaum \& Myer, 2012). Destacándose en importancia, acciones como la evaluación de la aptitud física y su posterior programación del ejercicio, en las escuelas y fuera de ellas. Esto no sucede en el sistema educativo argentino, ya que no existen programas que apliquen sistemáticamente evaluaciones de la condición física en los escolares (Secchi et al., 2014), con lo que se está perdiendo la oportunidad de percibir los niveles aptitudinales y los indicadores de riesgos futuros de los alumnos, información que resultaría de utilidad para la creación de estrategias que reviertan los porcentajes de inactividad física (Rodríguez et al., 2015).

La EF escolar, puede ser el espacio donde se empiecen a implementar evaluaciones relacionadas a la salud. Éstas aportan: motivación a los alumnos, conocimiento de sus niveles de aptitud física, promoción de las actitudes saludables e identificación de factores de riesgos, entre otros (Secchi et al., 2014).

La aptitud física a evaluar, entendida como el conjunto de elementos que incrementan la capacidad de trabajo (compuesta por la aptitud muscular, la flexibilidad, la composición corporal y la aptitud aeróbica), está fuertemente relacionada a los niveles de salud de los sujetos. En los últimos años ha ganado en visibilidad, a partir de su estudio, el rol de la aptitud muscular (compuesta por la fuerza máxima, 
explosiva, resistencia e isocinética) asociada con el bienestar físico. Ortega et al., (2012) muestran que bajos niveles de fuerza en la adolescencia, se asocian con los factores de riesgos clásicos y su posterior mortalidad prematura debido a enfermedades cardiovasculares. Asimismo, se confirmó una asociación inversa entre los niveles de aptitud muscular y los niveles de triglicéridos, LDLc, HDLc y glucosa en sangre. Dada la importancia de la aptitud muscular en la realización de las actividades diarias y ejercitaciones, es clave incluir dentro del currículo escolar espacios para evaluarla (Casas, 2016, Rodríguez et al., 2015).

Como la obesidad y el sobrepeso están fuertemente relacionados a los hábitos sedentarios, su testeo a partir de mediciones sencillas, arrojarían datos para dar una imagen integral de los evaluados y poder implementar acciones que colaboren con la reducción y prevención de la obesidad en adolescentes (Simmonds et al., 2016). La circunferencia de la cintura es un parámetro predictor del riesgo de enfermedad cardiovascular, y es una medida sencilla que se correlaciona bien con la grasa abdominal. Relacionado a esta medida, el índice cintura estatura (ICE) es un marcador de adiposidad en adolescentes. Los puntos identificativos de sobrepeso son 0,47 en niñas y 0,48 en niños, y los que señalan la obesidad son 0,50 y 0,51 respectivamente (Marrodán et al., 2013).

Los propósitos del estudio fueron: 1) Evaluar de manera sencilla la condición física de los alumnos, para la creación futura de acciones preventivas de la inactividad física. 2) Conocer y relacionar los niveles de aptitud muscular y adiposidad corporal, en los alumnos.

\section{METODOLOGÍA}

Este trabajo consiste en un pre-experimento investigativo cuantitativo, descriptivo y con diseño transversal. Se apeló al problema desde las técnicas de investigaciones cuantitativas, específicas para la recolección de datos, en busca del estudio de la aptitud muscular y la adiposidad corporal, en un único grupo de edad y sin seguimiento posterior. Las evaluaciones se realizaron en el marco de las clases de Educación Física Secundaria, bajo el tratamiento temático "Actividad física y salud". La unidad de análisis fue: adolescentes del tercer año de un colegio secundario privado de la ciudad de La Plata en el año 2017.

\section{Participantes}

La muestra del estudio fue de 17 alumnos ( $n=17)$, compuesta por $n=11$ varones y $n=6$ mujeres. Los participantes eran escolares pertenecientes a un tercer año de un colegio secundario privado de la ciudad de La Plata, con un promedio de edad de 14,92 £ 0,43 años, con un mínimo de 14, 41 y un máximo de 16,08. Es válido aclarar que este grupo se encontraba en plena etapa de pubertad, en la que suelen aparecer muchos cambios en el peso corporal, estatura y grasa corporal en ambos géneros.

\section{Criterios de inclusión y exclusión}

Los requisitos que debían cumplir los participantes para ser incluidos en el estudio fueron: ser alumnos del tercer año del secundario; haber recibido autorización médica al comienzo del ciclo lectivo, para realizar EF; presentar el consentimiento informado por escrito, el cual fue obtenido de los padres tras la explicación detallada del estudio, según los lineamientos de la OMS (2007). Fueron excluidos del estudio aquellos alumnos que no asistieron a alguna de las clases donde se llevaron a cabo las evaluaciones. 


\section{Toma de datos}

El total de las evaluaciones fueron realizadas en dos clases de EF, que se dictaban los días martes de 15:00 a 17:00 hrs. Durante la primera clase se evaluó a nueve participantes y en la siguiente, a los ocho restantes.

\section{Materiales e instrumentos}

-Estatura: se midió con un tallímetro autoadhesivo CALSIZE, el cual fue pegado en la superficie de la pared donde se realizaron las mediciones. Los alumnos estaban descalzos, con la columna vertebral apoyada en la pared y el mentón paralelo al piso, ubicando la cabeza en el plano horizontal de Frankfort (Casas, 2016). La medida se tomó en centímetros.

-Circunferencia de cintura prominente: se utilizó una cinta antropométrica metálica (CESCORF) para la realización de esta medición. La medida tomada en centímetros, se hizo en el punto medio entre el último arco costal y la cresta ilíaca (Casas, 2016).

-Índice cintura/estatura: para calcularlo se dividió la circunferencia de cintura por la estatura (Casas, 2016). Los participantes fueron asignados en tres grupos según valor de ICE (Marrodán et al., 2013): normopeso (menor a 0,47/0,48), sobrepeso (entre 0,47 y 0,49) y obesidad (mayor a 0,50), para poder establecer relaciones de cada grupo, con el desempeño muscular.

-Lanzamiento de balón medicinal: los alumnos se sentaron en el suelo con los pies delante de sus cuerpos, las rodillas dobladas y una bola de tres kilogramos delante de su pecho. Los jóvenes fueron instruidos para lanzar el balón medicinal (en un ángulo de $45^{\circ}$ ), 10 más hacia delante posible (Faigenbaum \& Mediate, 2006). La distancia del lanzamiento se midió en centímetros.

- Salto de Longitud sin carrera previa: fue evaluado como indicador de la fuerza de los miembros inferiores. Consiste en saltar con los pies juntos y con movimientos de brazos (sin carrera previa) la mayor distancia horizontal posible. La distancia alcanzada, tomada en centímetros, es la medida entre el talón del pie más atrasado y la línea de salida (Secchi et al., 2014, Ruiz et al., 2011).

-Trunk lift test (extensión de tronco): el objetivo de la prueba fue levantar la parte superior del cuerpo del suelo utilizando los músculos de la espalda y mantener la posición para permitir la medición. El participante yació sobre la colchoneta decúbito ventral, con los dedos de los pies apuntando hacia atrás, detrás del cuerpo y las manos colocadas debajo de los muslos. Cuando estuvo listo, el alumno levantó la parte superior del cuerpo del piso, de una manera muy lenta y controlada, hasta una altura máxima de 12 pulgadas $(30,48 \mathrm{~cm})$. La cabeza se mantuvo en línea recta con la columna vertebral. Una vez que se realizó la medición, el alumno regresó a la posición inicial. Se permitieron dos intentos, y se registró el mejor puntaje en centímetros (Plowman, 2013).

-FitnessGram Curl Up Test: el estudiante comenzó acostado de espaldas, con las rodillas dobladas a aproximadamente 140 grados, los pies planos sobre el piso, las piernas ligeramente separadas, los brazos estirados y paralelos al tronco con las palmas de las manos apoyadas en la colchoneta. Los dedos estaban extendidos y la cabeza en contacto con la colchoneta. La tira de medición se colocó en la estera debajo de las piernas de los estudiantes de modo que las yemas de los dedos descansaban en el borde más cercano de la tira de medición. Los pies no podían sostenerse contra un objeto. Manteniendo los talones en contacto con la colchoneta, los estudiantes se enroscaban lentamente, deslizando los dedos por la tira de medición hasta que las puntas de los dedos Ileguen al otro lado, luego se curvaban hacia abajo hasta tocar la colchoneta con la cabeza. El movimiento debe ser suave y a la cadencia de 20 flexiones por minuto (1 acurrucada cada 3 segundos). La cadencia curl-up, pitido para sentarse y acostarse, usa la misma cadencia descripta, se descargó para ser reproducido durante el desarrollo del test, mediante PC y amplificador de sonido (Plowman, 2013). Se cuantificaron las repeticiones de cada participante. 


\section{Procedimientos}

Antes de cada prueba, se dedicó un momento para enseñar a los alumnos la técnica apropiada de cada test y responder sus dudas. Además, se realizó una sesión de acondicionamiento previo, el cual consistió en 5 minutos de movimientos aeróbicos generales a intensidad baja, movilidad articular y elongación muscular. Todas las mediciones fueron realizadas con equipos idénticos y por el docente que supervisó todos los procedimientos. Cada prueba fue hecha según protocolo de test, en cuanto a repeticiones, toma de la mejor puntuación y medición en centímetros.

\section{Análisis estadístico}

Para el análisis estadístico de las variables estudiadas, se usó el programa SPSS (versión 22) y se aplicaron los criterios de Casas (2016). Se calcularon estadísticos descriptivos para la media y el desvío estándar de: estatura (E), circunferencia de cintura (CiC); índice cintura estatura (ICE); lanzamiento de balón medicinal (LBM); salto en longitud (SL); fuerza abdominal (FA); extensión de tronco (ET); índice aptitud muscular (IAM). Se utilizó el test de Student para determinar diferencias significativas entre las medias de las variables nombradas según sexo. Para la presentación de las relaciones estadísticas entre las variables continuas se aplicó el coeficiente de correlación de Pearson. Además, se construyó un índice de aptitud muscular en función de la suma de los puntajes z estandarizados de lo evaluado en: FA, SL, LBM y ET. En función de esto, se estableció una variable categórica con tres grupos: bajo (hasta el percentil 33), medio (entre el percentil 33 y 66) y alto (mayor al percentil 66).

\section{RESULTADOS}

A continuación, se muestran los resultados arrojados por el trabajo de campo realizado, guiado por el primer objetivo de este estudio: evaluar de manera sencilla la condición física de un curso del nivel escolar secundario.

Se resumen las características de la muestra en la Tabla 1 y se indican, los valores promedio „DS para las variables antropométricas y aptitud muscular. La prueba T de comparación de medias, mostró diferencias significativas en los valores de estatura $(\mathrm{p}=$ 0,009), SL ( $p=0,002)$, LBM ( $p=0,001)$ y el IAM ( $p=$ $0,029)$ a favor de los varones.

Tabla 1

Características de la muestra estudiada.

\begin{tabular}{|c|c|c|c|c|c|c|c|}
\hline \multirow{2}{*}{ Variable } & \multicolumn{2}{|c|}{ Total $(n=17)$} & \multicolumn{2}{|c|}{ Varones $(n=11)$} & \multicolumn{2}{|c|}{ Mujeres $(n=6)$} & \multirow{2}{*}{$\begin{array}{c}\text { Prueba } \mathrm{t} \\
\mathrm{p} \text { - valor }\end{array}$} \\
\hline & Media & DE & Media & DE & Media & DE & \\
\hline Estatura (en cm) & 164,29 & 7,44 & 167,54 & 6,19 & 158,33 & 5,88 & $0,009^{*}$ \\
\hline Circunferencia de cintura (en $\mathrm{cm}$ ) & 78,41 & 11,57 & 77,27 & 11,47 & 80,50 & 12,53 & 0,599 \\
\hline Índice cintura/estatura & 0,47 & 0.06 & 0,45 & 0,05 & 0,49 & 0,06 & 0,216 \\
\hline Lanzamiento de bola medicinal (en $\mathrm{cm}$ ) & 299,24 & 85,88 & 344,09 & 65,35 & 217,00 & 50,21 & $0,001^{*}$ \\
\hline Salto en largo (en cm) & 165,76 & 38,71 & 185,09 & 30,49 & 130,33 & 24,68 & $0,002^{*}$ \\
\hline Fuerza abdominal (en repeticiones) & 29,29 & 17,66 & 34,73 & 18,34 & 19,33 & 11,89 & 0,085 \\
\hline Extensión de tronco (en cm) & 29,64 & 2,73 & 28,90 & 3,20 & 31,00 & 0,00 & 0,137 \\
\hline Índice aptitud muscular & 0,00 & 2,78 & 1,06 & 2,87 & $-1,93$ & 1,17 & $0,029^{*}$ \\
\hline
\end{tabular}

*Diferencia estadísticamente significativa al $\mathrm{P}<0,05$

Para conocer y relacionar los niveles de aptitud muscular y adiposidad corporal de la muestra (segundo objetivo de este trabajo), se comparten los resultados arrojados por la investigación, en las siguientes tablas $(2,3$ y 4 ) y en la posterior Figura 1.
En la Tabla 2, se exponen las correlaciones entre las variables antropométricas y las de aptitud muscular. 
Tabla 2

Correlaciones de Pearson entre las variables relevadas en el estudio.

\begin{tabular}{|c|c|c|c|c|c|c|c|c|}
\hline Variable & Estatura & $\begin{array}{c}\text { Circunferencia } \\
\text { de cintura }\end{array}$ & $\begin{array}{c}\text { Índice } \\
\text { cintura/estatura }\end{array}$ & $\begin{array}{l}\text { Salto } \\
\text { en } \\
\text { largo }\end{array}$ & $\begin{array}{l}\text { Lanzamiento } \\
\text { de bola } \\
\text { medicinal }\end{array}$ & $\begin{array}{c}\text { Fuerza } \\
\text { abdominal }\end{array}$ & $\begin{array}{l}\text { Extensión } \\
\text { de tronco }\end{array}$ & $\begin{array}{l}\text { Índice } \\
\text { aptitud } \\
\text { muscular }\end{array}$ \\
\hline
\end{tabular}

Estatura $(\mathrm{en} \mathrm{cm}) \quad 1$

\begin{tabular}{|c|c|c|c|c|c|c|c|c|}
\hline $\begin{array}{l}\text { Circunferencia de } \\
\text { cintura (en cm) }\end{array}$ & 0,312 & 1 & & & & & & \\
\hline $\begin{array}{l}\text { Índice } \\
\text { cintura/estatura }\end{array}$ & 0,024 & 0955** & 1 & & & & & \\
\hline $\begin{array}{l}\text { Salto en largo (en } \\
\mathrm{cm} \text { ) }\end{array}$ & $0,524^{*}$ & $-0,464$ & $-0,648^{* *}$ & 1 & & & & \\
\hline $\begin{array}{l}\text { Lanzamiento de } \\
\text { bola medicinal } \\
(\mathrm{en} \mathrm{cm})\end{array}$ & $0,650^{* *}$ & 0,137 & 0,043 & $0,616^{* *}$ & 1 & & & \\
\hline $\begin{array}{l}\text { Fuerza } \\
\text { abdominal (en } \\
\text { repeticiones) }\end{array}$ & 0,169 & $-0,331$ & $-0,390$ & $0,680^{* *}$ & 0,406 & 1 & & \\
\hline $\begin{array}{l}\text { Extensión de } \\
\text { tronco }(\mathrm{en} \mathrm{cm})\end{array}$ & $-0,114$ & 0,334 & 0,296 & $-0,016$ & $-0,027$ & 0,197 & 1 & \\
\hline $\begin{array}{l}\text { Índice aptitud } \\
\text { muscular }\end{array}$ & $0,522^{*}$ & $-0,116$ & $-0,281$ & $0,829^{* *}$ & $0,715^{* *}$ & $0,819^{* *}$ & 0,426 & 1 \\
\hline
\end{tabular}

* La correlación es significativa al nivel 0,05 (bilateral)

** La correlación es significativa al nivel 0,01 (bilateral)

El ICE correlaciona positivamente con la CIC. EI SL correlaciona negativamente con el ICE. Es decir, al aumentar la distancia del SL, tiende a disminuir el ICE en nuestra muestra. Se trata de una correlación de -0,642 sustancial o media (Pérez, 2012). Esta asociación cuenta con un nivel de significación del 1\%. El lanzamiento de pelota medicinal correlaciona positivamente con la altura y con el salto en largo. El test de fuerza abdominal correlaciona positivamente y fuertemente con el SL. Por último, el índice de aptitud muscular correlacionó positivamente con la altura, SL, LBM y FT.
Sobre el total de los participantes, la distribución de la variable IAM señala un 29,2 \% (5) en grupo bajo, el 35,4\% (6) en el grupo medio y un 35,4\% (6) en el grupo alto.

La distribución de la variable categórica ICE, registró un 47,1\% (8) normopeso, el 23,5\% (4) se encuentra en sobrepeso y 29,4\% (5) en obesidad.

La siguiente Tabla 3, nos muestra la comparación entre las categorías normopeso, sobrepeso y obesidad en las variables de aptitud muscular. Los alumnos en categoría obesidad obtuvieron, en general, valores de medias menores en SL, LBM y FT.

Tabla 3

Cuadro de comparación de variables investigadas en los tres grupos según ICE.

\begin{tabular}{|c|c|c|c|c|c|c|c|c|c|c|c|c|}
\hline \multirow[t]{2}{*}{ Categoría } & \multicolumn{3}{|c|}{ Normopeso } & \multicolumn{3}{|c|}{ Sobrepeso } & \multicolumn{3}{|c|}{ Obesidad } & \multicolumn{3}{|c|}{ Total } \\
\hline & Media & $\mathbf{N}$ & $\begin{array}{l}\text { Desv. } \\
\text { Típ. }\end{array}$ & Media & $\mathrm{N}$ & $\begin{array}{c}\text { Desv. } \\
\text { Típ. }\end{array}$ & Media & $\mathrm{N}$ & $\begin{array}{l}\text { Desv. } \\
\text { Típ. }\end{array}$ & Media & $\mathrm{N}$ & $\begin{array}{l}\text { Desv. } \\
\text { Típ. }\end{array}$ \\
\hline Salto en largo & 186,13 & 8 & 33,732 & 178,00 & 4 & 20,331 & 123,40 & 5 & 21,755 & 165,76 & 17 & 38,719 \\
\hline Lanzamientos & 301,75 & 8 & 93,745 & 351,50 & 4 & 31,890 & 253,40 & 5 & 89,411 & 299,24 & 17 & 85,888 \\
\hline Flexión de tronco & 32,63 & 8 & 12,906 & 39,00 & 4 & 25,033 & 16,20 & 5 & 12,478 & 29,29 & 17 & 17,663 \\
\hline $\begin{array}{l}\text { Extensión de } \\
\text { tronco (máximo } \\
31 \mathrm{~cm} \text { ) }\end{array}$ & 29,000 & 8 & 3,1623 & 29,250 & 4 & 3,5000 & 31,000 & 5 &, 0000 & 29,647 & 17 & 2,7373 \\
\hline Suma de puntajes z & ,5088 & 8 & 3,12766 & 1,3300 & 4 & 2,58995 & $-1,8700$ & 5 & 1,49673 &, 0024 & 17 & 2,78602 \\
\hline
\end{tabular}


La Tabla 4, nos muestra la comparación entre Ios casos que pertenecen a un IAM bajo, medio o alto en cada variable de Aptitud muscular y el ICE. Coinciden los alumnos con IAM alto con una media ICE más baja que en las categorías baja o media.

Tabla 4

Cuadro de comparación de variables investigadas en los tres grupos según IAM.

\begin{tabular}{|c|c|c|c|c|c|c|c|c|c|c|c|c|}
\hline \multirow[t]{2}{*}{ Nivel IAM } & \multicolumn{3}{|c|}{ Bajo } & \multicolumn{3}{|c|}{ Medio } & \multicolumn{3}{|c|}{ Alto } & \multicolumn{3}{|c|}{ Total } \\
\hline & Media & $N$ & Desv. Típ. & Media & $\mathrm{N}$ & Desv. Típ. & Media & $\mathrm{N}$ & Desv. Típ. & Media & $\mathrm{N}$ & Desv. Típ. \\
\hline ICE &, 5000 & 5 & ,06403 &, 4714 & 7 & ,07335 &, 4400 & 5 & ,03674 &, 4706 & 17 & ,06280 \\
\hline Salto en largo & 127,40 & 5 & 27,373 & 168,14 & 7 & 31,035 & 200,80 & 5 & 20,462 & 165,76 & 17 & 38,719 \\
\hline Lanzamientos & 248,60 & 5 & 90,732 & 275,86 & 7 & 68,536 & 382,60 & 5 & 37,152 & 299,24 & 17 & 85,888 \\
\hline $\begin{array}{l}\text { Flexión de } \\
\text { tronco }\end{array}$ & 14,40 & 5 & 9,044 & 26,29 & 7 & 9,895 & 48,40 & 5 & 16,426 & 29,29 & 17 & 17,663 \\
\hline $\begin{array}{l}\text { Extensión de } \\
\text { tronco (máximo } \\
31 \mathrm{~cm} \text { ) }\end{array}$ & 27,800 & 5 & 4,4385 & 30,571 & 7 & ,7868 & 30,200 & 5 & 1,7889 & 29,647 & 17 & 2,7373 \\
\hline
\end{tabular}

En la Figura 1, se esquematizan las comparaciones de las tablas anteriores, entre las categorías del ICE y las variables de la aptitud muscular. En general, los alumnos que se encuentran en la categoría obesidad por tener un índice cintura estatura mayor a
0,51, tienen menores resultados en las pruebas de SL, LBM y FT. En la prueba de ET hay una paridad en las tres categorías según ICE. 

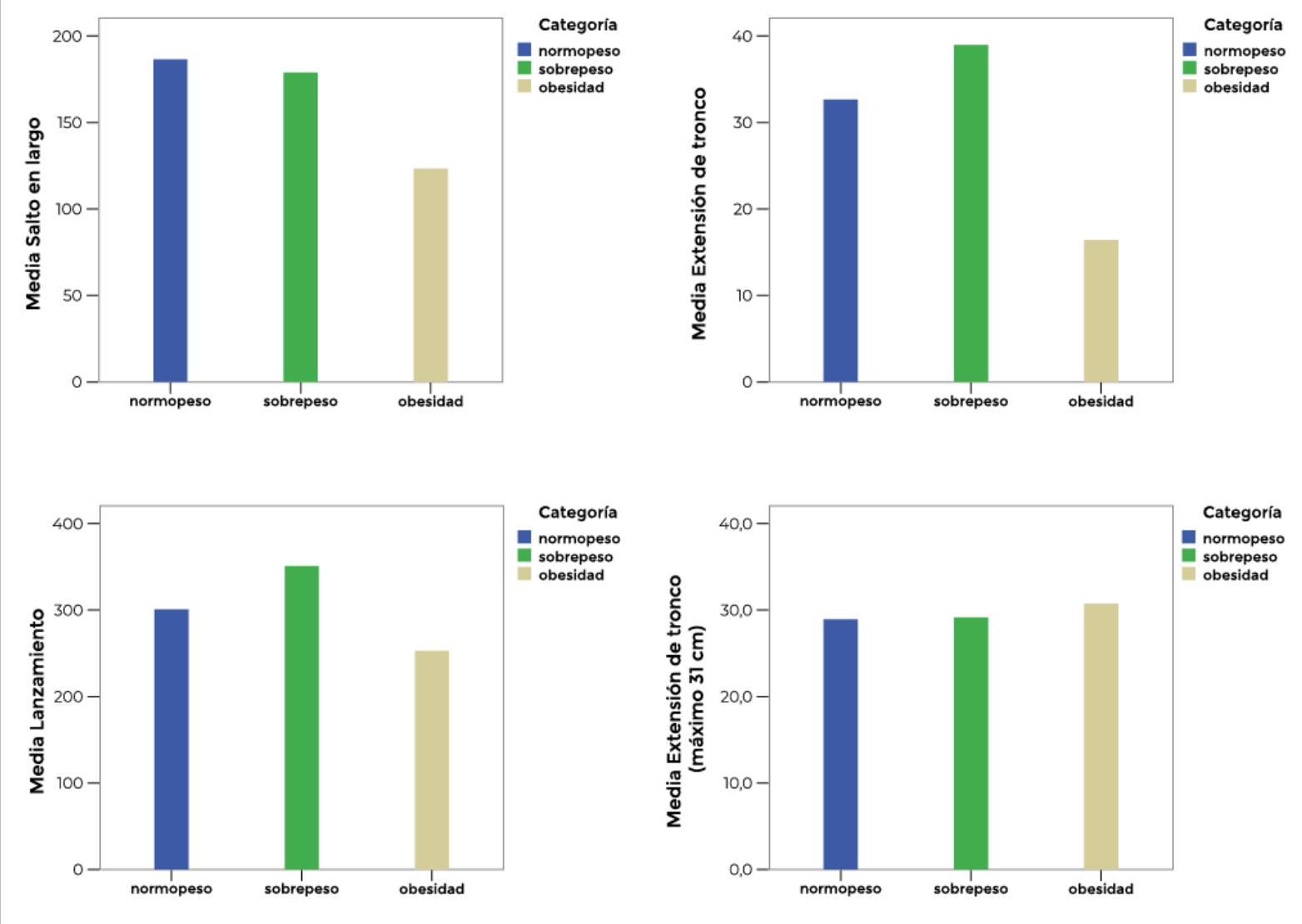

Nota: En la parte superior de la figura, se puede ver la comparación de la media en salto en largo (a la izquierda) y flexión de tronco (a la derecha) en los 3 grupos. En la parte inferior se comparan los resultados de la media de lanzamientos (a la izquierda) y la extensión de tronco (a la derecha).

Figura 7. Comparaciones del SL, FT, LBM y ET en los 3 grupos según el ICE.

\section{DISCUSIÓN}

Los resultados de este trabajo indican que se pudo evaluar de manera sencilla en el ámbito escolar, la condición física de los alumnos y se logró conocer y relacionar los niveles de aptitud muscular y adiposidad corporal. El SL correlaciona negativamente con el ICE (ver Tabla 2). Los casos que se encuentran en la categoría obesidad según ICE, obtuvieron resultados más bajos en las evaluaciones de la Aptitud muscular, a excepción del test de extensión de tronco (ver Tabla 3).

El primer objetivo de este trabajo se desprendía de la inquietud por proveer y aplicar herramientas sencillas para evaluación de la condición física en adolescentes de una institución escolar privada. Éste fue logrado, obteniendo información útil para la creación de futuras acciones preventivas sobre la inactividad física. En relación a esto, Secchi et al. (2014) reivindican la tarea docente, y Patiño \& Tabares (2018) afirman que estas acciones "ponen al profesor en Educación Física en un rol de promotor de la salud de sus alumnos" (p. 84).

Cabe aclarar que, en Argentina no hay antecedentes sobre una batería de evaluaciones de la condición física para la población por parte de instituciones promotoras de la salud. En este punto, sobresale la propuesta de Farinola et al. (2020), quienes crearon una batería de test para la población general, la cual brinda la posibilidad de avanzar en investigación, con datos certeros, actualizados y contextualizados. Aunque esta iniciativa esté orientada a la población adulta, es un progreso por la carencia de acciones en torno al sedentarismo y por la importancia 
que tiene para las políticas públicas en materia de salud. A propósito, la última Encuesta Nacional de Factores de riesgo (INDEC, 2019), realizada a la población urbana Argentina, con adultos de 18 años de edad (cuando finaliza la adolescencia), indica que la actividad física "escasa", aumentó y alcanzó a 6 de cada 10 individuos. Estos datos son negativos y concuerdan con la evidencia sobre esta epidemia (el sedentarismo) en América Latina. La mencionada encuesta, concluye sobre la necesidad de poner en marcha propuestas, como entornos escolares activos y el deporte en espacios comunitarios, a las que es necesario agregar, la evaluación estandarizada de la aptitud física en escolares y adultos.

Como segundo objetivo del trabajo, a partir de los test seleccionados, fue posible establecer relaciones estadísticas entre la aptitud muscular y el ICE. El salto en largo y el índice cintura- estatura se correlacionaron inversamente, es decir, al aumentar una variable, la otra disminuye. Según los resultados, en los casos que el SL fue mayor, tendió a coincidir con un ICE por debajo de la obesidad. Lo anterior concuerda con varios estudios, donde se informa que la aptitud muscular o neuromotora tiene relación con marcadores de la salud o factores de riesgos. Por ejemplo, el componente muscular de la aptitud física se relaciona inversamente con marcadores de la adiposidad (Ramírez et al., 2017). En estos estudios, también se incluyen el salto en largo como evaluación para la aptitud muscular y el ICE, como señalador de la adiposidad. Por lo tanto, se confirma la relación inversa entre la aptitud muscular y adiposidad corporal, concluyéndose que, un menor desempeño muscular, concuerda con un mayor riesgo de presentar exceso de tejido adiposo (Pacheco et al., 2016).

Los resultados de este estudio coinciden en que los adolescentes son cada vez más sedentarios, lo cual influye negativamente en su aptitud muscular adiposidad y bienestar general, aumentando así la prevalencia de factores de riesgos (Faigenbaum et al., 2013), ya que los alumnos caracterizados como obesos mediante ICE, obtuvieron menores resultados en las pruebas de aptitud muscular. Aquí, parece importante considerar los datos aportados por el estudio de Stricker et al. (2020), en el cual se revalida la importancia que tiene el entrenamiento de la resistencia muscular, en las clases de educación física en la escuela, ya que acarrea: mejoras en la salud, el estado físico y la composición corporal, la disminución de lesiones y también, aumentos en la aptitud cardiovascular, entre otros. Como punto a favor de este tipo de entrenamiento, la investigación confirma que niños inactivos consiguen una mayor adherencia al entrenamiento de la aptitud muscular (en comparación con los ejercicios aeróbicos).

\section{Fortalezas y limitaciones}

Una limitación de este trabajo es el tamaño de la muestra, consistente en $n=17 y$, además, la cantidad de mujeres fue menor $(n=6)$ que la de los varones $(n=11)$. Tal como en diversos estudios con diseño transversal, no se permiten establecer relaciones causales entre las variables analizadas. Sin embargo, se han realizado evaluaciones físicas y antropométricas validadas y utilizadas en cuantiosos trabajos científicos. Las evaluaciones y valoraciones fueron realizadas durante las clases de EF, fortaleciendo el rol de la asignatura en el espacio educativo, despertando el interés y motivación de los alumnos por mejorary aprender sobre la AF y la salud, proponiéndose, desde esta asignatura escolar, diferentes estrategias viables para el tratamiento de la situación epidemiológica, impactando en una población muy pequeña, pero que puede influir en otros para crear acciones en pos de la actividad física regular.

\section{CONCLUSIONES}

Se pudo evaluar satisfactoriamente, la condición física de los alumnos de un colegio secundario de la ciudad de La Plata, y se logró establecer la correlación inversa entre el SL y el ICE. El estudio muestra, en general, que a mayor adiposidad corporal, menor fuerza en los miembros inferiores. No se pudieron establecer relaciones con las otras pruebas (LBM, FT y ET). También es interesante resaltar que los alumnos categorizados como obesos (por su ICE) obtuvieron menores resultados en las pruebas de SL, FT $y\llcorner B M$. 
Para revertir la situación crítica en torno al sedentarismo, es importante avanzar en esta línea de investigación y continuar estudiando posibles acciones sencillas a llevar a cabo en la Educación Física escolar Argentina. Como también, la creación a nivel

\section{REFERENCIAS BIBLIOGRÁFICAS}

Carnero E., Alvero-Cruz, J., Giraldez, M., \& Sardinha, L. (2015). La evaluación de la composición corporal "in vivo"; parte I: perspectiva histórica. Nutrición Hospitalaria; 37(5), 1957-1967. https://doi.org/10.3305/nh.2015.31.5.8570

Casas, A. (2016). Análisis de las relaciones y efectos de los niveles de aptitud muscular sobre la coordinación motriz general y la adiposidad corporal en población escolar de 6 a 9 años [Tesis doctoral, Universidade Da Coruña, España]. http://hdl.handle.net/2183/17870

Faigenbaum, A., \& Mediate, P. (2006). Medicine Ball for All: A Novel Program that Enhances Physical Fitness in School-age Youths. Journal of Physical Education, Recreation \& Dance, 77(7), 1-58. https://www.researchgate.net/publication/ 233515649_Medicine_Ball_for_All_A_Novel_ Program_that_Enhances_Physical_Fitness _in_School-age_Youths.

Faigenbaum, A., \& Myer, G. (2012). Exercise deficit disorder in youth: Play now or pay later. Current Sports Medicine Reports, 17(4), 196200.

https://doi.org/10.1249/jsr.0b013e31825da961

Faigenbaum, A., Lloyd, R., \& Myer, G. (2013). Youth Resistance Training: Past Practices, New Perspectives, and Future Directions. Pediatric Exercise Science, 25(4), 591-604. https://doi.org/10.1123/pes.25.4.591

Farinola, M., Dardano, P., \& Maroni, G. (2020). Propuesta de evaluación de la condición física para población general: Batería Dickens. Educación Física Y Ciencia, 22(1), e114. https://doi.org/10.24215/23142561e114 nacional, de una batería de evaluaciones de la aptitud física y más específicamente de su componente, la aptitud muscular como marcador importante de la salud.

INDEC. Instituto Nacional de Estadística y Censos. (2019). $4^{\circ}$ Encuesta Nacional de Factores de Riesgo: resultados preliminares (1. ed.). Buenos Aires: INDEC, Secretaría de Gobierno de Salud. https://www.indec.gob.ar/ftp/cuadros/publicaciones/enfr_2018_resultados_definitivos.pdf

Malina, R. (2010). Physical Activity and Health of Youth. Ovidius University Annals, Series Physical Education \& Sport/Science, Movement \& Health, 10(2), 271-277. https://pdfs.semanticscholar.org/df09/dc68cff3b4388480d3fc42109a 3ccd952780.pdf

Marrodán, M., Martínez-Álvarez J., González-Montero de Espinosa, M., Lopez-Ejeda, N., Cabañas, M., \& Prado, C. (2013). Precisión diagnóstica del índice cintura-talla para la identificación del sobrepeso y de la obesidad infantil. Medicina Clínica, 140(7), 296-301. https://doi.org/10.1016/j.medcli.2012.01.032

Ministerio de Salud de la Nación argentina (2013). Tercera encuesta nacional de factores de riesgo para enfermedades no transmisibles. https://bancos.salud.gob.ar/sites/default/files/2018-10/0000000544cnt2015_09_04_encuesta_nacional_factores_riesgo.pdf

Nikolić, M., Jovanović, R., \& Stanković, A. (2020). Characteristics of physical activity among healthy serbian adolescents. Revista Brasileira de Medicina do Esporte, 26(1), 30-33. https://doi.org/10.1590/1517869220202601187598 
OMS. (2007). Recomendaciones para el consentimiento informado parental (OMS). Centro Interdisciplinario de Estudios en Bioética. Universidad de Chile https://www.uchile.cl/portal/investigacion/centro-interdisciplinario-de-estudiosen-bioetica/documentos/75657/documentos-de-consentimiento-informado-elaborados-por-la-oms

Ortega, F. B., Silventoinen, K., Tynelius, P., \& Rasmussen, F. (2012). Muscular strength in male adolescents and premature death: cohort study of one million participants. $B M J$, 345 , e7279. https://doi.org/10.1136/bmj.e7279

Pacheco-Herrera, J., Ramírez-Vélez, R., \& CorreaBautista, J. (2016). Índice general de fuerza y adiposidad como medida de la condición física relacionada con la salud en niños y adolescentes de Bogotá, Colombia: Estudio FUPRECOL. Nutrición Hospitalaria, 33(3), 556564. https://dx.doi.org/10.20960/nh.261

Patiño Palma, B., \& Tabares Gutiérrez, M. (2018). Determinantes sociales de la salud predictores de la condición física saludable en escolares entre 12 y 18 años en la ciudad de Dosquebradas Risaralda [Proyecto de Maestría, Universidad Autónoma de Manizales]. http://repositorio.autonoma.edu.co/handle/11182/146

Pérez Juste, R. (2012). Estadística aplicada a las Ciencias Sociales. Curso destinado a la preparación de futuros estudiantes de las asignaturas de Estadística de grados universitarios en CC. Sociales. Universidad Nacional de Educación a Distancia. https://www.intecca.uned.es/upload/noticias/20130614114556u_CURSOODEESTADISTICAAPLICADA.pdf

Plowman, S.A., \& Meredith, M.D. (Eds.). (2013). Fitnessgram/Activitygram Reference Guide (4th Edition). The Cooper Institute. https://www.cooperinstitute.org/vault/2440/web/files/662.pdf
Pradenas, X., Campos, M., Contreras, M., Puentes, D., \& Luna, P. (2017). Comparación del desarrollo motor en escolares de 9 y 10 años de edad en clases de educación física y talleres deportivos extracurriculares. Revista Ciencias de la Actividad Física UCM, 18(2), 1-8. https://doi.org/10.29035/rcaf.18.2.1

Ramírez Vélez, R., Cruz Salazar, S., Martínez, M., Cadore, E., Alonso Martínez, A., Correa Bautista, JE., ..., \& García Hermoso, A. (2017). Construct validity and test_retest reliability of the International Fitness Scale (IFIS) in Colombian children and adolescents aged 9_17.9 years: the FUPRECOL study. PeerJ 5, e3351. https://doi.org/10.7717/peerj.3351

Rodríguez Valero, F., Gualteros, J., Torres, J., Umbarila Espinosa, L., \& Ramírez-Vélez, R. (2015). Asociación entre el desempeño muscular y el bienestar físico en niños y adolescentes de Bogotá, Colombia. Nutrición Hospitalaria, 32(4), 1559-1566. https://doi.org/10.3305/nh.2015.32.4.9310

Ruiz, J. R., España Romero, V., Castro Piñero, J., Artero, E. G., Ortega, F. B., Cuenca García, M., ..., \& Castillo, M. (2011). Batería ALPHA-Fitness: test de campo para la evaluación de la condición física relacionada con la salud en niños y adolescentes. Nutrición Hospitalaria, 26(6), 1210-1214. https://scielo.isciii.es/scielo.php?script=sci_arttext\&pid=SO 212-16112011000600003

Secchi, J.D., García, G.C., España Romero, V., \& Castro Piñero, J. (2014). Condición física y riesgo cardiovascular futuro en niños y adolescentes argentinos: una introducción de la batería ALPHA. Archivos Argentinos de Pediatría, 172(2), 132-140. http://doi.org/10.5546/aap.2014.132

Simmonds, M., Llewellyn, A., Owen, C. G., \& Woolacott, N. (2016). Predicting adult obesity from childhood obesity: a systematic review and meta-analysis. Obesity Reviews, 17(2), 95107. https://doi.org/10.1111/obr.12334 
Stricker, P., Faigenbaum, A., McCambridge, T., \& COUNCIL ON SPORTS MEDICINE AND FITNESS (2020). Resistance Training for Children and Adolescents. Pediatrics, 745(6), e20201011. https://doi.org/10.1542/peds.2020-1011

Tuñon, I., \& Laíño, F. (2014). Insuficiente actividad física en la infancia: niños, niñas y adolescentes entre 5 y 17 años en la Argentina urbana [en línea] Serie del Bicentenario 20102016; boletín n². Observatorio de la Deuda Social Argentina. Barómetro de la Deuda Social de la Infancia. Universidad Católica Argentina. https://repositorio.uca.edu.ar/bitstream/123456789/8132/1/i nsuficiente-actividad-fisica-infancia.pdf
US Dept of Health and Human Services. (2008). Physical Activity Guidelines for Americans. https://health.gov/sites/default/files/201909/paguide.pdf

World Health Organization (2010). Global Recommendations on Physical Activity for Health. https://www.who.int/es/publications/i/item/9789241599979

\section{Dirección para correspondencia}

Pablo Ariel Torres

Universidad Nacional de La Plata.

La Plata, Argentina

ORCID ID: https://orcid.org/0000-0002-2334-6114

Contacto:

patunlp@gmail.com

Recibido: 14-01-2021

Aceptado: 03-08-2021 